\title{
Gastric stimulation in treatment in type 2 diabetes mellitus
}

\author{
Zonca $\mathrm{P}^{1,2}$, Hoppe $\mathrm{C}^{2}$, Cambal $\mathrm{M}^{2,3}$, Jacobi $\mathrm{CA}^{2}$ \\ Department of Surgery, University and University Hospital Ostrava, Czech Republic. pavel.zonca@fno.cz
}

\begin{abstract}
Metabolic surgery is a dynamic field providing a wide range of new techniques. The aim of our paper is to inform about gastric electrostimulation in the treatment of type 2 diabetes mellitus. Gastric electrostimulation in type 2 diabetes mellitus treatment is performed by means of implanting Tantalus system (Metacure). When triggered by food intake, the Tantalus system generates signals for the stomach to contract. Early after the food intake, thus before the stomach distends completely, the system increases spontaneous stomach contractions. Via afferent vagal signals, these increased contractions are evaluated by the central nervous system as a sign that satiety has been reached. Three pairs of electrodes connected with a stimulator are implanted laparoscopically. The system comes equipped with a battery as well.

Laparoscopically implanted system of stomach electrostimulation presents a simple and easily regulated system without disturbing the integrity of gastrointestinal tract. It is a reversible system. In accord with preliminary results, Tantalus offers a safe and effective treatment of type 2 diabetes mellitus by reducing other cardiometabolical risky factors. The operation safety is comparable with that of similar minimally invasive surgical techniques. In order to improve our understanding of diabetes mellitus pathophysiology as well as of effects of gastric electrostimulation, more trials need to be performed. The surgical diabetes treatment represents an interesting chance for patients and it might become a common technique in the future (Fig. 1, Ref. 29). Text in PDF www.elis.sk. Key words: diabetes mellitus, obesity, gastric stimulation, pacemaker.
\end{abstract}

Overweight and obesity contribute to the development of diabetes mellitus. It is a global problem. Some authors use a slightly exaggerated expression "globesity". The care provided to patients with diabetes mellitus is the domain of internal medicine. The dynamically developing metabolic surgery offers an efficient treatment to selected patients. In the same way that the bariatric surgery has been established as a method of obesity treatment (1), the methods applied in bariatric surgery have been promoted in the treatment of metabolic syndrome. The indications for surgery in these terms are shifting to patients with BMI $<35 \mathrm{~kg} / \mathrm{m}^{2}$. BMI is going to be a secondary parameter of surgery indication. The priority lies in the treatment of metabolic syndrome. There are several surgical possibilities of treating the metabolic syndrome. The authors present the gastric electrostimulation as a technique aimed at treating mellitus treatment.

\section{Methods}

The system of gastric electrostimulation in the obesity and metabolic syndrome treatment is based on the following principle. The intake of meal induces stomach distension, which in turn increases stomach contractility. Under normal physiological

\footnotetext{
${ }^{1}$ Department of Surgery, University and University Hospital Ostrava, Czech Republic, ${ }^{2}$ Center for Visceral and Miniinvasive Surgery, Wesseling, Germany, and ${ }^{3}$ Department of Surgery, Faculty of Medicine, Comenius University, Bratislava, Slovakia

Address for correspondence: P. Zonca, MD, PhD, Department of Surgery, University and University Hospital Ostrava, 17. listopadu 1790, CZ-708 52 Ostrava-Poruba, Czech Republic.
}

conditions, this reaction sends afferent vagal signals leading to the feeling of satiety. An animal trial with rats under laboratorial conditions confirmed that non-excitatory antral electric stimulation increases the amplitude of antral contraction and stimulates the vagal afferent signals in the central nervous system similarly as when triggered by passive distension with meal (2). The canine trials confirmed that chronic gastric electrostimulation leads to a reduction in food intake, as well as to weight loss with no changes in contractility and gastric evacuation (3).

The Tantalus system used to be originally dedicated solely to obesity treatment. Currently it is used in treatment of type 2 diabetes mellitus. A typical meal of west population can be possibly described as being hypovolemic and hypercaloric. This type of meal increases the prevalence of obesity and metabolic syndrome. It takes longer for a hypovolemic and hypercaloric meal to activate the mechanical activity of the stomach and induce the feeling of satiety than it takes for high-volume meal which leads to earlier stomach distension and satiety. Theoretically the fact that the system of gastric electric stimulation could shorten the time needed for the feeling of satiety to be aroused could help also the patients with diabetes mellitus. The other mechanism functions via motoric stimulation, which improves the neurohumoral control of glycemia through direct effect in the bowel, and mediates the metabolic changes in the remote tissues. The centers in central nervous system are activated as well. These centers contribute to glucose, insulin and glucagon regulations.

The Tantalus system (Metacure NV) is an implantable system that mediates the signal of stomach contraction triggered by food intake. This system synchronizes the stomach's own contractile 


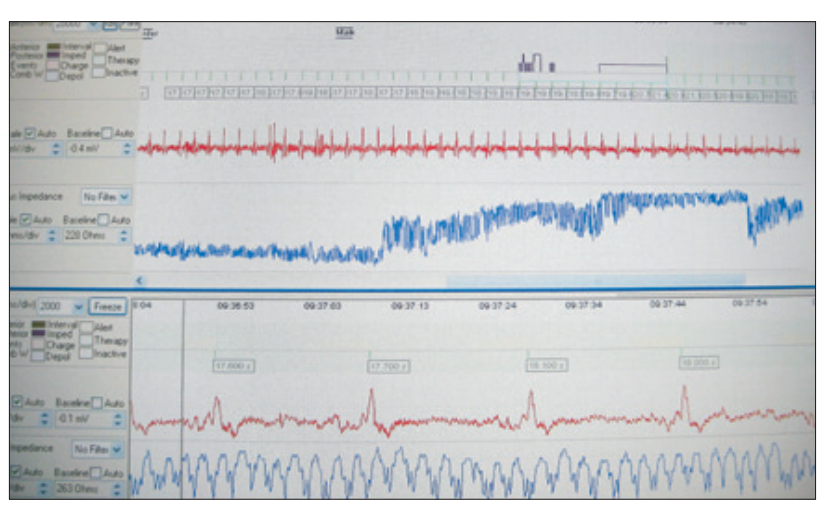

Fig. 1. Change in impedance after food intake and synchronous increase in motoric activity.

activity with the stimulated activity. It produces a discontinuous signal initiated by food intake. The increased spontaneous gastric contraction early after the food intake, yet before the stomach becomes fully distended, leads to the sensation of satiety produced via afferent vagal signals sent into the central nervous system. Taking into consideration that the generated signals are triggered by natural electric activity, the system does not interfere with normal frequency of slow waves in the antrum. The amplitude of motoric activity increases (Fig. 1) (4). Tantalus system consists of a pulse generator with three bipolar electrodes and a battery. The first electrode pair (Ultraflex, Metacure Ltd.) is implanted in the ventral fundus, the second one in the ventral antrum, and the third one in the dorsal antrum. The electrodes in the fundus are placed $2 \mathrm{~cm}$ lateral from the gastro-esophageal junction. Both electrodes are located in parallel along the long stomach axis and are $2 \mathrm{~cm}$ apart from each other. The electrodes in the fundus detect the stomach distension after food intake. The electrodes in the ventral and dorsal regions of antrum are placed about 2 and $4 \mathrm{~cm}$ orally from the antrum. The axis of electrodes is transverse to the long axis of stomach. The electrodes in the antrum detect the slow waves from the antrum, and in addition, they are able to send a synchronized signal from the pulse generator. The electrodes are placed beneath the serous membrane. The system is implanted laparoscopically. The subserosal electrode placement is intra-operatively checked by a flexible gastroscope. Direct eye control helps to avoid the intraluminal placement of electrodes. The electrodes are attached by two clips distally and proximally to the stomach wall by means of non-absorbable stitch. The electrodes are connected with a pulse generator, which is implanted above the fascia in the left mesogastrium. The battery is placed under the ribs on the left side. The system functioning is tested during the surgery. Six weeks after the implantation, the activation of the system follows. Initially, the function of detecting the food intake as well as the ability of detecting the spontaneous electrical antrum activity is evaluated. The detection of food is considered successful when it takes place five minutes after the beginning of food intake (defined meal).

An optimal patient for System Tantalus is a moderately obese patient with type 2 diabetes mellitus not able to control his con- dition sufficiently with peroral antidiabetic drugs, is a potential candidate for insulin treatment, and is either insufficiently compliant with the treatment or treatment recommendations in respect of diabetes or the latter treatment has adverse side effects.

About 200 systems with Tantalus have so far been implanted worldwide. The first implantation was performed in Vienna. The most of the patients with this system are in Austria, Germany, and France. Recently, some patients in Warsaw have been also treated in this way. The exclusion criteria for implantation are as follows: previous implantation of pulse generator, previous bariatric surgery, instable weight within four weeks of observation, antidepressive treatment, food intake disorder (bulimia, etc.), obesity caused by endocrine disorder, use of slimming pills within the past three months, and disorders of gastrointestinal motility. The most optimal indication for implantation relates to patients with type 2 of diabetes mellitus with BMI of $30-35 \mathrm{~kg} / \mathrm{m}^{2}$. It is an important fact that type 2 diabetes mellitus candidates for treatment with Tantalus have to have at least a partially maintained insulin secretion.

\section{Discussion}

The treatment of diabetes and its complications requires a complex multi-disciplinary approach. For many reasons, the treatment with diet and peroral antidiabetic agents is often insufficient. In many cases, the insulin treatment offers just a temporary solution to this problem. The serious complications are brought about when the treatment of diabetes is insufficient. Diabetes mellitus is connected with an increased risk of cardiac or cerebral stroke, development of renal insufficiency, eye problems, and in $60 \%$ of cases, with non-traumatic amputations of lower extremities (5).

One of the possible ways of treating diabetes mellitus relies on application of the methods of bariatric surgery. In the early 1980's, many surgeons reported cases of complete remission in diabetic patients after gastric by-pass performed due to obesity (6). Many of the trials demonstrated the possibility of curing diabetes after the bariatric surgery. Buchwald concluded that after the gastric by-pass, the remission of diabetes took place in $98.9 \%$ of patients with biliopancreatic diversion or duodenal switch. The remission after gastric by-pass was observed in $83.7 \%$, and after the gastric bandage, in $47.9 \%$ (7-10). Diabetes control or remission was better achieved after intestinal bypass (gastric bypass or biliopancreatic diversion). The purely restrictive operations improve the diabetes control but are not as effective as bypass operations. The identical effect of diabetes treatment is achieved also in the non-obese population. Many trials with patients with BMI $<35 \mathrm{~kg} / \mathrm{m}^{2}$ have been performed. In his trial, Lee showed that in $89.5 \%$ of patients with diabetes with BMI $<35 \mathrm{~kg} / \mathrm{m}^{2}$, the remission was achieved one year after the gastric bypass (11).

The effect of bariatric surgeries is explained through the lower food intake, weight loss, sugar and fat malabsorption, and alteration of bowel regulation hormones. It is known that glycemic control after surgery improves usually within days or weeks after surgery, i.e. before weight loss is achieved. About one-third of patients after gastric bypass achieve normoglycemia and are free of antidiabetic medication before being dismissed. This dia- 
betes compensation is not associated with weight reduction. It is the improvement in sensitivity to insulin that triggers directly an improvement in the function of $\beta$-cells in the pancreas (12-19). The hormonal diversion is achieved. The induction of glucagonlike peptides, namely GLP-1 and GLP-6 is achieved as a result of non-digested food coming in the aboral parts of the gastrointestinal tract. The ghrelin level is influenced as well. This effect generates pro-insulin (20). There are two theories. The first is a theory of upper gastrointestinal tract (foregut-hypothesis) and the second one is a theory of lower gastrointestinal tract (hindgut-hypothesis). The theory of upper GI tract is based on the thesis that the elimination of duodenal receptors and receptors in proximal part of GI tract from the passage interferes with a still unknown factor, which plays a crucial role in the development of insulin resistance in type 2 of diabetes mellitus. The theory of low GI tract says that the fast movement of food in the distal part of lower GI tract leads to better metabolism of glucose owing to the secretion of GLP-1 and other hormones. GLP-1 is produced by L cells in the distal part of bowel. GLP-1 stimulates insulin secretion and enforces a proliferative and anti-apoptic effect on the pancreatic $\beta$-cells. Rubino performed a trial on rats with diabetes mellitus by means of malabsorptive operation. After the surgery, remission in diabetes was achieved, and after the reversal operation, the rats redeveloped diabetes again. Rubino's trial clearly confirmed the benefit of gastrointestinal bypass in type 2 diabetes mellitus (21). Both theories involve neurohumoral mechanisms including the signals integrated in the central nervous system. It is assumed that chemical and electric cascades could be mediated through vagal afferent nerves. It is necessary to assume that the stimulation in one part of the bowel leads to a mechanical or biochemical response in other segments.

The duodeno-jejunal bypass belongs among other operations influencing the metabolic syndrome. Providing we had a better knowledge of physiology and pathophysiology of the gastrointestinal tract, the intestinal transposition could be applied more extensively (22).

Surgical intervention influences hyperuricemia, hypertriglyceridemia, non-alcoholic liver steatosis, hypertension, and cardiovascular complications.

Gastric electrostimulation belongs among the latest methods of influencing the glucose regulation. A clinical trial confirmed a weight loss of $4.5 \mathrm{~kg}$ and reduction in Hbalc by $0.5 \% \pm 0.3 \%$ $(p=0.05)$ in obese patients with diabetes mellitus one year after the surgery. In the subpopulation of patients remaining on a stable or reduced oral anti-diabetic medication, a weight loss of 6.3 $\mathrm{kg}$ and Hbalc reduction by $0.9 \% \pm 0.3 \%(\mathrm{p}=0.05)$ was achieved (23). Patients with an efficient response to gastric electric stimulation were identified as well as those with minimal response to electrostimulation.

Another treatment method is represented by vagal blockade. This technique was described by Camilleri in 2008. A high-frequency alternating electric current impulse generated by an implanted generator blocks the branches of vagal nerve. The electrodes are placed near the vagal nerves in the gastroesophageal junction (24).
Another treatment option is that of endoscopic implantation of duodenal stent, which eliminates the duodenal receptors. The stent imitates the situation as seen in duodeno-jejunal bypass. The food passes through the duodenum without coming in contact with mucosa. The aboral parts receive poorly digested food $(25,26)$.

The technique of gastric electrostimulation using the described Tantalus system represents a borderline between the conservative therapy and radical bypass operations. It could be an appropriate choice for patients refusing surgery that disturbs the integrity or continuity of gastrointestinal tract. It is a completely reversible operation. Bariatric surgery is commonly considered to be a highrisk surgery. In this connection Buchwald presented an interesting meta-analysis. This meta-analysis summarizes 361 trials with 85,048 patients with early mortality of $0.28 \%$ (30 days) and late mortality of $0.35 \%$ (30 days till 2 years) (27). This mortality is comparable to that in patients with laparoscopic cholecystectomy $(0.26-0.6 \%)$. Despite this fact, the cholecystectomy is considered to be a routine and safe surgery contrary to bariatric surgery, which is considered to be extremely dangerous $(28,29)$.

Metabolic surgery allows achieving a new aim in diabetes mellitus treatment. This aim has been shifted from mere controlling the symptoms to achieving complete remission. The methods applied in the bariatric and metabolic types of surgery have contributed to better understanding of physiology and pathology of diabetes mellitus.

The economy should be considered as well. The surgery costs including the peri-operative care are relatively high, but according to the economic analysis, the conservative therapy brings higher expenses 2-3 years later. In the long run, the surgery is unambiguously a less expensive option and a more beneficial alternative for patients.

\section{Conclusion}

The metabolic surgery is a young and dynamically developing field. It is based on the observation that diabetes could be treated with incretins by increasing the sensitivity to insulin. Patients with implanted Tantalus system are observed not only in terms of influencing the diabetes mellitus, but also in terms of cardiometabolic risk factors. Obesity, hypertension, insulin resistance, inflammatory reaction, coagulation cascade, and fat metabolism are evaluated. The gastric electrostimulation influences the neural, hormonal and cellular functions of the gastrointestinal tract. The future will show the clinical efficiency of this system. As opposed to the fate of Tantalus in Greek mythology, the patients with Tantalus system are not tantalized with hunger and thirst. In order to gain a better understanding of pathophysiology of diabetes and electric stimulation effects, more trials need to be performed.

\section{References}

1. Fried M, Hainer V, Basdevant A, Buchwald H, Dietel M, Finer N, Greve JW, Horber F, Mathus-Vliegen E, Scopinaro N, Steffen R, Tsigos C, Weiner R, Widhalm K. Interdisciplinary European guidelines on surgery for severe obesity. Rozhl Chir 2008; 87 (9): 468-476. 
2. Yin J, Chen JD. Implantable gastric electrical stimulation: ready for prime time? Gastroenterology 2008; 134: 665-667.

3. Eagon JC, Kelly KA. Effect of electrical stimulation on gastric electrical activity and emptying. Neurogastroenterol Motil 1995; 7: 39-45.

4. Bohdjalian A, Prager G, Aviv R, Policker S, Schindler K, Kretschmer S, Riener R, Zacherl J, Ludvik B. One-year Experience with Tantalus ${ }^{\mathrm{TM}}$ : a New Surgical Approach to Treat Morbid Obesity. Obesity Surg 2006; 16: 627-634.

5. National diabetes fact sheet: general information and national estimates on diabetes in the United States, 2003, Centers for Disease Control and Prevention, Ed. Atlanta, GA, U.S. Department of Health and Human Services, Centers for Disease Control and Prevention, 2004.

6. Pories WJ. Diabetes: the evolution of a new paradigm. Ann Surg 2004; 239: $12-13$.

7. Pories W, MacDonald KJ, Morgan E, Sinha MK, Dohm GL, Swanson MS, Barakat HA, Khazanie PG, Leggett-Frazier N, Long SD, O'Brien KF, Caro JF. Surgical treatment of obesity and its effect on diabetes: 10-y follow-up. Am J Clin Nutr 1992; 55 (Suppl 2): 582S-585S.

8. Schauer PR, Burguera B, Ikramuddin S, Cottam D, Gourash D, Hamad G, Eid GM, Mattar S, Ramanathan R, Barinas-Mitchel E, Rao RH, Kuller L, Kelley D: Effect of laparoscopic Roux-en Y gastric bypass on type 2 diabetes mellitus. Ann Surg 2003; 238: 467-485.

9. Buchwald H, Avidor Y, Braunwald E, Jensen MD, Pories W, Fahrbach $\mathbf{K}$, Schoelles K. Bariatric surgery: a systematic review and metaanalysis. JAMA 2004; 292: 1724-1737.

10. Sjostrom L, Lindroos AK, Peltonen M, Torgerson J, Bouchard C, Carlsson B, Dahlgren S, Larsson B, Narbro K, Sjöström CD, Sullivan M, Wedel H. Swedish Obese Subjects Study Scientific Group: Lifestyle, diabetes, and cardiovascular risk factors 10 years after bariatric surgery. N Engl J Med 2004; 351: 2683-2693.

11. Lee W, Wang W, Lee Y, Huang MT, Ser KH, Chen JC. Effect of laparoscopic mini-gastric bypass for type 2 diabetes mellitus: comparison of BMI $>35$ and $<35 \mathrm{~kg} / \mathrm{m}^{2}$. J Gastrointest Surg 2008; 12: 945-952.

12. Cummings DE, Overduin J, Shannon MH, Foster-Schubert KE. Hormonal mechanisms of weight loss and diabetes resolution after bariatric surgery. Surg Obes Relat Dis 2005; 1 (3): 358-368.

13. Pories WJ, Swanson MS, MacDonald KG et al. Who would have thought it? an operation proves to be the most effective therapy for adultonset diabetes mellitus. Ann Surg 1995; 222 (3): 339-352.

14. Schauer PR, Burguera B, Ikramuddin $S$ et al. Effect of laparoscopic Roux-en Y gastric bypass on type 2 diabetes mellitus. Ann Surg 2003; 238 (4): 467-485.

15. Service GJ, Thompson GB, Service FJ, Andrews JC, CallazoClavell ML, Lloyd R. Hyperinsulinemic hypoglycemia with nesidioblastosis after gastric bypass surgery. N Engl J Med 2005; 353 (3): 249-254.
16. Patti ME, McMahon G, Mun EC et al. Severe hypoglycemia postgastric bypass requiring partial pancreatectomy: evidence for inappropriate insulin secretion and pancreatic islet hyperplasia. Diabetologia 2005; 48 (11): 2236-2240.

17. Bantle JP, Ikramuddin S, Kellogg TA, Buchwald H. Hyperinsulinemic hypoglycemia developing late after gastric bypass. Obes Surg 2007; 17 (5): 592-594.

18. Cummings DE. Gastric bypass and nesidioblastosis - too much of a good thing for islets? N Engl J Med 2005; 353 (3): 300-302.

19. Cummings DE, Weigle DS, Frayo RS et al. Plasma ghrelin levels after diet-induced weight loss or gastric bypass surgery. N Engl J Med 2002; 346 (21): 1623-1630.

20. Weiner RA. Indikation und Prinzipien der metabolischen Chirurgie. Der Chirurg 4, 2010: 379-395.

21. Rubino F, Forgione A, Cummings DE, Vix M, Gnuli D, Mingrone G, Castagneto M, Marescaux J. The mechanism of diabetes control after gastrointestinal bypass surgery reveals a role of the proximal small intestine in the pathophysiology of type 2 diabetes. Ann Surg 2006; 244 (5): 741-749.

22. Cohen RV, Schiavon CA, Pinheiro JS, Luiz Correa J, Rubino F. Duodenal-jejunal bypass for the treatment of type 2 diabetes in patients with body mass index of $22-34 \mathrm{~kg} / \mathrm{m}^{2}$ : a report of 2 cases. Surg Obes Relat Dis 2007; 3: 195-197.

23. Bohdjalian A, Prager G, Rosak C, Weiner R, Jung R, Schramm M, Aviv R, Schindler K, Haddad W, Rosenthal N, Ludvik B. Improvement in Glycemic Control in Morbidly Obese Type 2 Diabetic Subjects by Gastric Stimulation. Obes Surg 2009; 19: 1221-1227.

24. Camilleri M, Dokuli J, Herrera M. Intra-abdominal vagal blockin (VBLOC therapy): clinical results with a nex implantable medical device. Surgery 143: 723-731.

25. Tarnoff M, Shikora S, Lembo A. Acute technical feasibility of an endoscopic duodenal-jejunal bypass sleeve in a porcine model: a potentially novel treatment for obesity and type 2 diabetes. Surg Endosc 2008; 22: $772-776$.

26. Gersin KS, Keller JE, Stefanidis D. Duodenal-jejunal bypasse sleeve: a totally endoscopic device for the treatment of morbid obesity. Surg Obnov 2007; 14: 275-278.

27. Buchwald H, Estok R, Fahrbach K, Banel D, Sledge I. Trends in mortality in bariatric surgery: a systematic review and meta-analysis. Surgery 2007; 142: 621- 632 (discussion 632-635).

28. Rubino F, Moo TA, Rosen DJ, Dakin GF, Pomp A. Diabetes surgery: a new approach to an old disease. Diab Care 2009; 32 (Suppl 2): S368-372.

29. Baltasar A, Serra C, Pérez N, Bou R, Bengochea M, Ferri L. Laparoscopic sleeve gastrectomy: a multi-purpose bariatric operation. Obes Surg 2005; 15: 1124-1128. 